\title{
Progress of Chromium Migration and Transformation in Groundwater- Rock Interface
}

\author{
Y.ZOU\&H.Z.Zhang \\ School of Water Resource and Environment, China University of Geosciences, Beijing, China
}

Keywords: Groundwater-rock interface, Chromium, Transportation and Transformation, Models.

\begin{abstract}
Chromium migration and transformation is a complex process in groundwater-rock interface, including a series of physical and chemical reactions, such as adsorption-desorption, redox, dissolution-precipitation, complexation-dissociation etc. It was affected by groundwater $\mathrm{pH}$, Eh, the type and the content of dissolved substances, the velocity of groundwater, the lithology of aquifer medium and other factors. This paper reviews the chromium migration and transformation in groundwater-rock interface, migration models and simulation softwares involved in this process.
\end{abstract}

\section{Instructions}

Water-rock interaction is a geochemistry process between water and rock (rock and mineral), it attracts the attention of researchers in earth science, environmental chemistry, hydrogeology, engineering geology, etc. Since 1970s, water rock interaction began to involve in the pollution and remediation of groundwater. Domestic and foreign scholars carried out lots of research on migration and transformation of the organic pollutants, variable valence heavy metals and nitrogen, phosphorus in water-rock interface, and provides an important theoretical basis for the prevention and control of pollution.

In recent years, with the rapid development of the industry involved in chromium and chromium chemicals widely used, unsaturated zone and groundwater pollution have become increasingly serious caused by chromium compounds into the groundwater system. Chromium disposition and distribution in groundwater-rock interface is a complex process, influenced by many factors, such as forms of chromium, types and quality of groundwater, physical and chemical properties of aquifer, etc. The study on migration and transformation of chromium in groundwater-rock interface has an important guiding significance for repairing and controlling the chromium pollution of groundwater.

\section{Migration and Transformation of Chromium in Groundwater-rock Interface}

Existing forms of chromium in groundwater determine the chromium migration and transformation capabilities, and its distribution between solid phase and liquid phase. In the natural groundwater and unsaturated zone, chromium usually exists as two forms: Cr (VI) and Cr (III). Main Existing Forms of $\mathrm{Cr}(\mathrm{VI})$ are $\mathrm{Cr}_{2} \mathrm{O}_{7}{ }^{2-}, \mathrm{CrO}_{4}{ }^{2-}, \mathrm{HCrO}_{4}{ }^{-}$, with the negatively charged(Sun, 2011). Small portion of $\mathrm{Cr}$ (VI) can be adsorbed to soil colloids and formed insoluble substance with soil component, while most are free in groundwater. $\mathrm{Cr}$ (III) mainly exists as $\mathrm{Cr}^{3+}$ and $\mathrm{CrO}^{2-}$, which easily occurred adsorption and complexation with soil colloids and organic matter, or generate oxygen and hydroxide precipitate, with poor mobility. Cr (VI) and Cr (III) can be transformed into each other by redox in appropriate circumstances. Therefore, chromium reaches equilibrium by adsorption-desorption, oxidation-reduction, precipitation-dissolution, complexation-dissociation process in groundwater-rock system.

Adsorption-Desorption.In the Eh-pH range of the natural groundwater-rock system, Cr (VI) mainly exists in the form of oxyacid anion. The adsorption of Cr (VI) by soil colloid is the primary way that it migrates from liquid into solid. There are mainly two types of adsorption: physical adsorption and physicochemical adsorption. In acidic soil of $\mathrm{pH}<6, \mathrm{Cr}$ (VI) is absorbed as the form of $\mathrm{HCrO}_{4}{ }^{-}$, which belongs to the electrostatic adsorption (Liu,2000). Clay minerals with inorganic 
hydroxide groups including iron oxide, alumina, montmorillonite, kaolinite and fewer, as the main adsorption of anions, control the adsorption quantity of anions( $\mathrm{Lu}, 2007)$, accompanying by hydroxyl releasing. Soil colloids can also produce specific adsorption by exchanging with $\mathrm{OH}^{-}$and $\mathrm{HCrO}_{4}^{-}$, which has the stronger adsorption force. The adsorption of $\mathrm{Cr}$ (VI) is mostly physicochemical adsorption.

The soil $\mathrm{pH}$ has some significant effect on the adsorption of $\mathrm{Cr}$ (VI). One is the soil $\mathrm{pH}$ can change the chemical form of $\mathrm{Cr}$ (VI) so that effect on the content of $\mathrm{HCrO}_{4}^{-}$. $\mathrm{Li}$ Hangbin(Li,2011)found that, the content of $\mathrm{HCrO}_{4}{ }^{-}$decreased as $\mathrm{pH}$ increased, while the content of $\mathrm{CrO}_{4}{ }^{2-}$ increased as $\mathrm{pH}$ increased. When the $\mathrm{pH}$ is larger than 8, there only $\mathrm{CrO}_{4}{ }^{2-}$ exists in the system. The adsorption has almost come to a halt. On the other side, the soil $\mathrm{pH}$ can change the surface charge of soil minerals (Yan, 2010). Lower $\mathrm{pH}$ is helpful to the apparent adsorption of $\mathrm{Cr}$ (VI).The positive charge on the hydrated metal oxides surface decreased and negative charge increased with the rise in $\mathrm{pH}$, so that the adsorption of $\mathrm{Cr}$ (VI) decreased.

Compared with other anions, such as chloride ions, nitrate ions and sulfate ions, $\mathrm{Cr}$ (VI) is absorbed to oxides and clay particles more tightly, which was slightly inhibited when the Cr (VI) concentration was higher. The results of competitive adsorption of anions show that, competing oxyanions promoted $\mathrm{Cr}$ (VI) desorption, directly participating in competition of adsorption site, while when the concentration of $\mathrm{SO}_{4}{ }^{2-}, \mathrm{F}^{-}$and $\mathrm{H}_{2} \mathrm{PO}_{4}{ }^{-}$reached $10^{-4} \mathrm{~mol} / \mathrm{L}$, there has a significant inhibitory effect (Zhang,1990). Many scholars' research (Zhu,1985) showed that different minerals have different $\mathrm{Cr}$ (VI) adsorption capacity. Its order is kaolinite> illite> montmorillonite. Red soil dominated by kaolinite, containing a large number of iron and aluminum oxide colloids with positive charge, with weak negative and low $\mathrm{pH}$, has strong adsorption of Cr (VI) (Xiong,1975). While the yellow brown soil mainly illite and the chernozem mainly montmorillonite on Cr (VI) adsorption is relatively weak, the order of $\mathrm{Cr}$ (VI) adsorption capacity of three kinds of soil is red soil > yellow brown soil > chernozems.

Soil clay minerals in Cr (III) adsorption ability is 30 300 times larger than Cr (VI). Cr (III) adsorption process and capacity depends on the water-rock system $\mathrm{pH}$, and increases with the rise of $\mathrm{pH}$. When the groundwater $\mathrm{pH}$ is relatively low, Cr (III) exists as complex cation, such as $\mathrm{CrOH}_{2}{ }^{+}, \mathrm{Cr}(\mathrm{OH})_{2}{ }^{+}$; while at high $\mathrm{pH}$, exists as complex anion, such as $\mathrm{Cr}(\mathrm{OH})_{4}^{-}(\mathrm{Li}, 2005)$. In the range of $\mathrm{pH} 2 \sim 6$, Cr (III) cation was mainly nonspecifically adsorbed by soil colloid with the negative charge. $\mathrm{Cr}$ (III) cations may also be nonspecifically adsorbed by desorption or association of $\mathrm{H}^{+}$with hydroxyl in the colloid group (i.e., physicochemical adsorption). In $\mathrm{pH} 4 \sim 6$, $\mathrm{Cr}$ (III) is easily adsorbed and co-precipitated with $\mathrm{Fe}^{3+}$ and $\mathrm{Al}^{3+}$ (Liu,2000;Li,2005). Research (Luan,1990) shows that changes of water oxidation reduction potential have a great impact on the release of heavy metals from sediments. The order is the oxidation state $>$ weak oxidation $>$ reduction state. If the state is relatively stable, the lower oxidation reduction potential is, the more easily the heavy metal released. The reason is that manganese oxides be reduced easily make oxide colloid partial dissoluted, thus reducing the amount of adsorption of heavy metals. At the same time, the exchange adsorption of $\mathrm{Cr}$ (III) of active components in the soil can transfer Cr (III) from water to soil. Haiyan Sheng (Sheng,2005) proved that there are many functional groups on the humus, such as $\mathrm{COOH},-\mathrm{NH}_{2}$, which can generate a complex chelate reaction with $\mathrm{Cr}$ (III), so that the $\mathrm{Cr}$ (III) is strongly adsorbed. The order of Cr (III) adsorption capacity of different kinds of minerals is: montmorillonite > illite > kaolinite (He,2001) The research of Chen Yingxu(Chen,1994) showed that at pH 4, Qingzini predominated montmorillonite has strong electronegativity, the Cr (III) adsorption amount is large; dry red soil and veins mud field dominated with kaolinite and iron and aluminum oxides has weak electronegative, thus has relatively small adsorption capacity. Liu Yunhui(Liu,2000) shows that in the same condition of pH, Cr (III) adsorption ability of brown soil is larger than fluvo aquic soil and cinnamon soil.

Oxidation-Reduction.Because the toxicity and mobility of $\mathrm{Cr}$ (VI) are far larger than $\mathrm{Cr}$ (III), it is expected that $\mathrm{Cr}$ (VI) can be directly transformed into $\mathrm{Cr}$ (III) that reduce its harm under reducing conditions. Therefore, the study of the oxidation-reduction reaction of chromium in the soil mainly focusses on two aspects in recent years. One is how $\mathrm{Cr}$ (VI) be reduced to $\mathrm{Cr}$ (III) when it gets into 
the soil, and if the Cr (III) in the reaction product can be reduced again; the other is the reaction behavior of these reactions in different conditions. At the same time, another focus is the addition of reducing substances to promote the reduction of $\mathrm{Cr}$ (VI).

$\mathrm{Cr}$ (VI) can transform into $\mathrm{Cr}$ (III) in four ways: the reduction reaction with $\mathrm{H}_{2} \mathrm{~S}, \mathrm{SO}_{2}$ and $\mathrm{Fe}^{2+}$, electron-transfer reactions occurs on the mineral surface, the reaction with non humic organic matter (such as carbohydrates and protein) and the reduction reaction with soil humus(Wang,2007). In the general range of $\mathrm{pH}$ and Eh, Cr (VI) will be reduced by organic compounds with hydroxyl, ferrous ions and the soluble sulfide soon. The lower the $\mathrm{pH}$, the faster the reduction reaction(Li,2005;Eary,2007).

Inseong Hwang(Hwang,2002) demonstrated that the reduction capability of $\mathrm{Fe}^{2+}$ in the solution can be partly transferred to solid medium that the solid phase medium can continue to remove $\mathrm{Cr}$ (VI) through the research of the influence of $\mathrm{Fe}^{2+}$ on the reduction kinetics of $\mathrm{Cr}(\mathrm{VI})$ in aquifer, and proved that it is feasible method by injecting $\mathrm{Fe}^{2+}$ in the aquifer to remedy $\mathrm{Cr}$ (VI) contaminated aquifer and groundwater. Kh rici-Bousnoubra (Kh, 2009) discovered that the $\mathrm{H}_{2} \mathrm{~S}$ released by industrial site get into the shallow layer, reducing the $\mathrm{Cr}$ (VI) to insoluble $\mathrm{Cr}$ (III). Lirong Chen (Chen,2011) demonstrated that when the organic matter content is greater than $2 \%$ in the soil, $\mathrm{Cr}$ (VI) will almost all be reduced to Cr (III), generating insoluble hydroxide precipitation so that be adsorbed and fixed. In the acid condition, purifying effect of humic acid on Cr (VI) can be attributed to: firstly $\mathrm{Cr}_{2} \mathrm{O}_{7}{ }^{2-}$ diffuse to humic acid in the solution, and be adsorbed and redoxed, $\mathrm{Cr}$ (III) in reduction product partly be adsorbed, and part of it get into the water again.

More than $90 \%$ of $\mathrm{Cr}$ (III) compounds in soil can be quickly fixed, however, Cr (III) adsorbed by clay minerals in soil continuous desorb affected by soil $\mathrm{pH}$ and the oxidant, due to the role of ligand such as $\mathrm{Cl}^{-}, \mathrm{SO}_{4}{ }^{2-}, \mathrm{HCO}_{3}{ }^{-}$returns to the water $(\mathrm{Gao}, 2005)$ and small part of $\mathrm{Cr}$ (III) can be oxidized to $\mathrm{Cr}$ (VI). Cr (III) oxidation is prone to occur in the soil with rich organic matter and poor $\mathrm{MnO}_{2}$ (Garman,2004), therefore, it is necessary to consider the content of manganese oxide in soil or sediments when predicting the formation and harm of chrome material, but Cr (III) in the water can hardly be oxidized by the oxygen in the air(Lai,2006). Yingxu Chen(Chen,1993) consider that $\mathrm{Cr}$ (III) diffuse from the solution to the surface of $\mathrm{MnO}_{2}$ particles so that occur complexation reaction, and transfer electron with $\mathrm{MnO}_{2}$, as a result, $\mathrm{Cr}$ (III) is reduced to Cr (VI) anionic and entre the water. Hongmin Yang(Yang,2009) found that in the acidic environment, $\bullet \mathrm{OH}$ can be oxidant of $\mathrm{Cr}(\mathrm{III}), \mathrm{Cr}(\mathrm{VI})$ increased with increasing concentration of $\bullet \mathrm{OH}$.

$\mathrm{Cr}$ (VI) reduction process is influenced by $\mathrm{pH}$. Junxiang Yang's (Yang,2005) research on the impact of $\mathrm{pH}$ on the $\mathrm{Cr}(\mathrm{VI})$ reduction by $\mathrm{S}^{2-}$ indicates that the higher the $\mathrm{pH}$, the smaller the reaction rate. It is probably due to in neutral and alkaline conditions, $\mathrm{S}^{2-}$ can be oxidized by dissolved oxygen. Jiaquan $\mathrm{Xu}(\mathrm{Xu}, 1994)$ believes that the general trend of the effect of $\mathrm{pH}$ on reaction rate constant $\mathrm{K}$ is that $\mathrm{K}$ increases with the rise of $\mathrm{pH}$. In $\mathrm{Fe}^{2+}$ system, $\mathrm{Cr}$ (VI) reduction reaction will stop at $\mathrm{pH}>8.0$, which is because $\mathrm{Fe}^{2+}$ occurs precipitation. Xue Liu's(Liu,2010) experiments indicate that the electromotive force of $\mathrm{Cr}(\mathrm{VI})$ reduction $\left(\mathrm{E}^{\theta}=1.23\right)$ in the acidic condition is larger than that in the alkaline conditions. Therefore, it can be concluded that $\mathrm{Cr}$ (VI) reduction reaction is easier in low $\mathrm{pH}$. Hellerich(Hellerich,2005) also confirmed that when $\mathrm{pH}$ is less than 4, about $50 \%$ of $\mathrm{Cr}$ (VI) are reduced to $\mathrm{Cr}$ (III); but in neutral water, $\mathrm{Cr}$ (VI) concentration and total chromium are similar. Therefore, $\mathrm{Cr}$ (VI) compounds are only existing in neutral or weak alkaline environment (Sun, 2007).

Soil redox potential determines the form and concentration of chromium and the main mineral elements in system (Su,2010). The higher the redox potential, the stronger the oxidation ability, the more easily the Cr (III) oxidation reaction occurs, and the more stable the Cr (VI) exist. When the redox potential is relatively low, Cr (III) forms insoluble compounds with sulfide easily, being fixed by clay minerals or closed by iron and aluminum oxides.

Precipitation- Dissolution.Cr (VI) compound solubility is large in general, but it can precipitate with barium, lead, silver and other heavy metal ions (Zhao,2006).However, Due to low concentrations of these metal, $\mathrm{Cr}(\mathrm{VI})$ in water has strong ability.

Cr(III) precipitation include oxide, hydroxide and chromium dihydrogen phosphate. Due to the 
presence of alkaline substances, carbonate and other $\mathrm{pH}$ buffer substances in the soil and groundwater, and the solubility product of $\mathrm{Cr}(\mathrm{OH})_{3}$ is small $\left(\mathrm{K}_{\mathrm{SP}}=5.4 \times 10^{-31}\right)$, which make most of the $\mathrm{Cr}$ (III) transfer from groundwater to soil as $\mathrm{Cr}(\mathrm{OH})_{3} \bullet \mathrm{nH}_{2} \mathrm{O}$ (Dong,2006). In the water-rock system, Cr (III) began to precipitate at $\mathrm{pH}>4$, at the range of $\mathrm{pH} 4 \sim 6$, Cr (III) precipitation increased with $\mathrm{pH}$, and at $\mathrm{pH}$ 5.5, Cr (III) almost all precipitated. (Sun,2010; Yang,2011). This precipitation is ligand molecules of $\mathrm{Cr}$ (III) and water or hydroxyl. In the strong alkaline water of $\mathrm{pH}>10.5$, Cr (III) hydrolysis occurs, generating $\mathrm{Cr}(\mathrm{OH})_{4}{ }^{-}$ion, precipitated $\mathrm{Cr}$ (III) partly transfer into water again, at this time, the soil colloid is also with strong negative charged and $\mathrm{Cr}(\mathrm{OH})_{4}{ }^{-}$is free(Chen,1994) in liquid phase. In the range of $\mathrm{pH} 3 \sim 7$, phosphate anions which mainly dominated by $\mathrm{H}_{2} \mathrm{PO}_{3}{ }^{-}$can precipitate with $\mathrm{Cr}$ (III) to generate $\mathrm{Cr}\left(\mathrm{H}_{2} \mathrm{PO}_{4}\right)_{3}$ precipitation in the water so that $\mathrm{Cr}$ (III) concentration decreased rapidly, the existence of phosphate to $\mathrm{Cr}$ (III) pH rapidly decreased critical precipitation. But the presence of phosphate has little effect on $\mathrm{Cr}$ (III) hydrolysis.

pH has a significant effect on the solubility of Cr (III). Changxun Dong (Dong,2006)considered that the rise of $\mathrm{pH}$ leads to $\mathrm{Cr}$ (III) hydrolysis degree enhanced that form $\mathrm{Cr}(\mathrm{OH})_{3}$ precipitate, which can improve the stability of Cr (III). Bin Yang (Yang,2006) believed that, at low pH, Cr (III) can form organic complexes, so that the migration ability is improved; at $\mathrm{pH}>4$, Cr (III) solubility reduced, and completely precipitated at $\mathrm{pH}$ 5.5. Cr (III) could form polycyclic compounds of chromium, and ultimately precipitated as chromium hydroxide in alkaline water. Kun Zhao(Zhao,2006) found Cr (III) can be adsorbed by hydrated iron, aluminum oxide on the surface to be the component of the crystal in weak acidic or alkaline conditions, thus forming the coprecipitation.

Other ions in the water-rock system can be co-precipitated with Cr (III). Huifang Sun(Sun,2010) found that in the presence of organic matter, the existence of $\mathrm{AlCl}_{3}$ and $\mathrm{FeCl}_{3}$ makes a contribution to the removal of $\mathrm{Cr}$ (III) from the water, and in most cases, the co-precipitation of $\mathrm{AlCl}_{3}$ is better than $\mathrm{FeCl}_{3}$. Xiuli Yang(Yang,2011)proved that the hydrolysis of calcite in Ma Lan loess (i.e. carbonate) can cause the Cr (III) co-precipitation reaction by simulation and experiment of waterrock interaction.

Complexation-Dissociation. Complexation is a complicated process of metal ions and inorganic or organic ligands (Ding,1998). Complex and free ion are in a balanceable and dynamic state, which has significant influence on the adsorption. In natural water, $\mathrm{Cr}$ (VI) generally will not generate coordination compounds, while inorganic ligands and organic with negative charged form stable complexes with $\mathrm{Cr}$ (III).

Cr (III) can form complexes with hydroxyl, ammonia, fluoride, cyanide, thiocyanate, bromide ion, sulfate and other inorganic ligands. Guo Wang (Wang, 1994) argues that these inorganic ligands affect adsorption by complexing with Cr(III). Jingsheng Chen(Chen,1975) believes that the complexation of hydroxyl on heavy metal is actually the hydrolysis reaction of heavy metal ions, improving the solubility of heavy metal hydroxides greatly. In recent years, the complexation of organic matter and Cr (III) become the focus of research, including the complexation ability of different kinds of organic matter, the mechanism of complexation reaction, the stability of complex, influencing factors of complexation, etc. There are hydroxyl, carboxyl, enolate, sulfonic acid, amino and many other important complexing functional groups and chelating groups in the organic matter of the soil. Complexing functional groups can provide electronic, generating metal-organic complexes with metal ion, while chelating groups have more than two electronic ligands that forming chelating. Yajun Wang(Wang,2007) believes that the humic acid containing many functional groups, with large amount of negative charge, so that the adsorption of heavy metal cations is high, but considering that the surface of soil colloids are also negatively charged, the humic acid only combined with chromium ion to change the valence of the chromium ions, in order to enhance the interaction between chromium ion and soil particles. Jiang Jiang(Jiang,2001) holds that the complexation of the dissolved organic matter and heavy metal is prone to form soluble complexes, enhancing the solubility of heavy metals. Huifang Sun (Sun,2010) proved that EDTA is a ligand with six teeth that has the strongest chromium complexing ability by studying on the 
removal of $\mathrm{Cr}$ (III) from water under the existence of a variety of organic compounds respectively; in addition, citric acid easily form complexes with chromium due to providing 3 matching site.

\section{The Simulation}

The mathematical models used to describe the dynamic characteristics of chromium migration and transformation are usually Langmuir equation, the Temkin model, Henry model, the first order dynamic model, the second-order kinetic equation, the Elovich equation etc. At present, much research carried by domestic and foreign scholars show that the Freundlich equation, Langmuir equation can simulate chromium adsorption process more properly in the soil.

Yan Feng(Yan,2008) believes that the soil pH of Cr (VI) have a greater impact on apparent adsorption dynamics. The first order kinetic equation and parabolic model can better characterize the $\mathrm{Cr}$ (VI) characteristics of the adsorption kinetics in acidic soil, correlation coefficient was 0.99, while these equations cannot extend to alkaline soil. Ailing Ren (Ren,2000) and UweBuczko(UweBuczko,2004) preliminarily analyze the mechanism and adsorption dynamic of chromium migration and transformation in unsaturated zone through the soil column leaching experiment and numerical simulation, found that the isothermal adsorption process of $\mathrm{Cr}$ (III), Cr (VI) both accorded with Langmuir equation. About the chromium reduction and precipitation reaction, most studies have demonstrated that the reduction between $\mathrm{Cr}$ (VI) and sulfide is the first order reaction. But the research of Junxiang Yang(Yang,2005) on the reaction kinetics and the factors of $\mathrm{Cr}(\mathrm{VI})$ and $\mathrm{S}^{2-}$ in solution at constant temperature and anaerobic conditions showed that the initial reaction stage is the first order reaction in the constant solution $\mathrm{pH}(7 \sim 9)$ and excessive sulfide, the reaction rate was significantly increased at the late reaction.

In recent years, research on simulation has been paid more attention to. The software applied widely are numerical simulation software Visual-Modflow and geochemical simulation software Phreeqc. Visual Modelflow is applied to similate the migration process of pollutants in the groundwater, widely used in the actual field, but rarely used in chromium migration research in domestic and abroad. While Phreeqc can choose some equations describing the corresponding chemical reactions, calculating the composition of multiphase equilibrium solution. Jeanine (Jeanine,2002) applied the Phreeqc software to simulate chemical equilibrium to predict the concentrations of various ions in chromium slag leachate and the variation of $\mathrm{pH}$, which showed that $\mathrm{Cr}$ (VI) in chromium slag exist in chromium calcium aluminate (sandwiched layered double hydroxides with chromium) and hydrogarnet containing Cr (VI), solid phase dissolved lead to the dissolution of $\mathrm{Cr}$ (VI), calcium, aluminum, silicon and magnesium in the range of $\mathrm{pH} 8$ 12. Under natural conditions $\mathrm{pH}$ is unlikely to change much in the landfill due to high $\mathrm{pH}$ buffer capacity of chromium slag. Xiuli Yang (Yang,2011) analyze the component, surface structure, surface site concentrations and cation exchange capacity and other characteristics of Malan loess, according to the result of analysis, Phreeqc was utilized to establish the water-rock interaction model of Malan Loess and $\mathrm{Cr}$ (III), and carried out the variable initial concentration experiment, removal of carbonate experiment and extraction experiment, which illustrate that the removal of $\mathrm{Cr}$ (III) by Malan loess in aqueous solution are mainly chromium precipitation and co-precipitation caused by the hydrolysis of calcite and surface chromium complexation of manganese oxide, supplemented by chromium cation exchange of silicate minerals.

\section{Conclusions}

Cr transfers in the groundwater-rock interface through adsorption and desorption, oxidation and reduction, precipitation and dissolution and complex and dissociation,which influenced by groundwater $\mathrm{pH}$, Eh, organic matters, other coexisting ions and the properties of the aquifer,etc. Due to the complexity of several reactions, existing at the same time and difficulty in distinguishing, and different features of migration and transformation in different soil and water, it's an extremely difficult assignment to determine the chromium behavior of migration and transformation., It is necessary to Improve the knowledge of chromium behavior in water-rock interface to accurately determine the potential crisis, and also can improve the method to reducing 
the danger of pollution area to the lowest level, adopt effective methods to repair and governance unsaturated zone and underground water contaminated by chromium.

At present, most research on chromium contaminating in groundwater-rock interface stay in the stage of laboratory simulation, lack of combination of aquifer of actual contaminated sites and groundwater characteristics, and the research of a plurality of effect factors is not enough, that is urgent to carry out the simulation research of the actual site . Revealing the mechanism of chromium pollution in water-rock interface with a number of interactions, establishing pollution model of the actual sites is conducive to predict chromium contaminated site pollution more accurately, so that the control of chromium pollution in groundwater has much more pertinence.

\section{References}

[1] BuczkoU \& Hopp L \& Berger W, et al. 2004. Simulation of chromium transport in the unsaturated zone for predicting contaminant entries into the groundwater, Journal of Plant Nutrition and Soil Science, 167(3):284-292

[2] Chen, Lirong \& Chen, Xiaoluo \& Yan, Zhihui. 2011. Migration and transformation of heavy metal pollutants in the soil and the treatment of chromium. Science and Technology Innovation Herald, (33):122-123

[3] Chen, Jingsheng. 1975. Migration of Heavy Metals in the Environment, Environmental Science, (5):50-55

[4] Chen, Yingxu \& Luo, Yongming \& Zhu, Yongguan, et al. 1994. Study on Chemical Behavior of Chromium in Soils V. Factors Affecting Cr(III) Adsorption and Precipitation in Soils, Acta Pedologica Sinica, 31(01):77-85.

[5] Chen, Yingxu \& He, Zengyao \& Zhu, Zuxiang. 1993. Mechanism of $\mathrm{Cr}(\mathrm{m}$ ) Oxidation by Mn Oxioes. Acta Scientiae Circum stantiae, 13(1):45-50.

[6] Deakin D \& West L J \& Stewart D I, et al. 2001. Leaching behaviour of a chromium smelter waste heap, Waste Management, 21(3):265-270.

[7] Ding, Aizhong. 1998. Studies of Mechanics and Treatment of Groundwater Pollution from Landfill. Chinese Academy of Geological Sciences.

[8] Dong, Changxun \& Pan, Genxing \& Lan, Yeqing. 2006. Influence of solution pH and preadsorption of ions on oxidation of $\mathrm{Cr}(\mathrm{III})$ by $\mathrm{MnO} 2$ crystals. Ecology and Environment, 15(1):2731.

[9] Eary L E \& Davis A. 2007. Geochemistry of an acidic chromium sulfate plume. Applied Geochemistry, 22(2):357-369.

[10] Gao, Hongge \& Li, Baiying \& Chen, Lihui, et al. 2002. Chromium Migration in Soil and Groundwater and Method for Removal of Chromium in Groundwater. North environment, (01):3032.

[11] Garman S M \& Luxton T P \& Eick M J.2004. Kinetics of chromate adsorption on goethite in the presence of sorbed silicic acid, Journal of environmental quality, 33(5):1703-1708.

[12] Geelhoed J S \& Meeussen J C \& Hillier S, et al. 2002. Identification and geochemical modeling of processes controlling leaching of $\mathrm{Cr}$ (VI) and other major elements from chromite ore processing residue, Geochimica et Cosmochimica Acta, 66(22):3927-3942.

[13] Hellerich L A \& Nikolaidis N P. 2005. Studies of hexavalent chromium attenuation in redox variable soils obtained from a sandy to sub-wetland groundwater environment. Water research, 39(13):2851-2868.

[14] He, Hongping \& Guo, Jiuhao. 2001. An Experimental Study of Adsorption Capacity of Montmorillonite, Kaolinite and Illite for Heavy Metals, Acta Petrologica et Mineralogica, 
20(4):573-578.

[15] Hwang I \& Batchelor B \& Schlautman M A. 2002. Effects of ferrous iron and molecular oxygen on chromium(VI) redox kinetics in the presence of aquifer solids, Journal of Hazardous Materials, B92 (2002):143-159.

[16] Jiang, Jiang \& Wang, Guo \& Fang, Ling. 2001. Complexation between soil water-soluble organic matter and heavy metal. Soil and Environmental Sciences , 10(01):67-71.

[17] Kh E Rici-Bousnoubra H \& Kh E Rici N \& Derradji E F, et al. 2009. Behaviour of chromium VI in a multilayer aquifer in the industrial zone of Annaba, Algeria. Environmental geology,57(7):1619-1624.

[18] Lai H \& Mcneill L S. 2006. Chromium redox chemistry in drinking water systems. Journal of Environmental Engineering-ASCE, 132(8):842-851.

[19] Li, Hangbin. 2011. The Study of the dissolution-release of $\mathrm{Cr}(\mathrm{Vl})$ and translocation model in soil in chromium slag. Chang Sha: Central South Univ

[20] Li, Zhiping \& Qu, Jihong \& Shen, Zhaoli, et al. 2005. Simulation Experimental Study of Comparison of the Removal of Cr(VI) by Different Aquifer Medium. Geotechnical Investigation\& Surveying, 4:19-22.

[21] Li, Zhiping \& Chen, Xiaogang \& Shen, Zhaoli. 2006. Influence on shallow ground water by chromium in polluted river. Acta Scientiae Circum stantiae , 26(1):99-104.

[22] Liu, Xue \& Wang, Xingrun \& Zhang, Zengqiang. 2010. Potential influences of $\mathrm{pH}$ and organic matter on the occurrence forms of chromium in chromium-contaminated soils. Chinese Journal of Environmental Engineering, (6):1436-1440.

[23] Liu, Yunhui \& Wei, Xianyou. 2000. A Study on the Adsorption of Chromium in Soil and the Form Extraction, Journal of Agricultural Universityof Hebei,23(1):16-20.

[24] Lu, Peng \&Wu, Xiaofu \& Yu, Xubiao, et al. 2007. Simulation of the Kinetic Process of Chromium (VI ) Adsorption in Liquid/Solid Systems, Journal of Central South University of Forestry \& Technology (Natural Science),27(1):118-123.

[25] Luan, Zhaokun \& Tang Hongxiao.1990. Study on the Chemical Stability of Heavy Metals in polluted water. Environmental Science , 11(4):18-25.

[26] Ren, Ailing \& Guo, Bin \& Liu, Sanxue, et al. 2000. Study on the Migration of Chromium in Soil. Urban Environment\& Urban Ecology, 13(02):54-56.

[27] Sun, Jun. 2007. Catalysis of $\mathrm{Al}(\mathrm{III})$ and $\mathrm{Fe}(\mathrm{III})$ on $\mathrm{Cr}(\mathrm{VI})$ Reduction by Organic Acid. Nanjing: Nanjing Agricultural University.

[28] Sheng, Haiyan. 2005. Preliminary studies of Cr(III) absorbing capacity in Qinghai soil of development from loess. Journal of Qinghai University (Nature Science), 23(6):35-38.

[29] Sun, Chao \& Tian, Xizhao \& Shan, Qiang, et al. 2011. The Experimental Study on Chromium VI Pollution Abatement of Site Soil, China Population, Resources and Environment, 21(3):93-96.

[30] Su, Changqing. 2010. Study on Microbial Reduction of Cr (VI) and the Stability of Cr(III) in contaminated soils. Chang Sha, Central South University.

[31] Sun, Huifang \& Zhang, Ruihua \& Shi, Yulei. 2010. The Removal of Chromium from Water in the Presence of Organic Compounds, Technology of Water Treatment, 36(07):46-49.

[32] Wang, Yajun \& Zhu, Kun \& Wang, Jinxi, et al. 2007. Study on the humic acid adsorption of chromium in sandy soil. Journal of Safety and Environment, 7(05):42-47.

[33] Wang, Guo. 1994. Effects of heavy metal ion adsorption by complexation, Progress in Soil 
Science, 22(06):6-13.

Xiong, Yi.1975. Association pattern and mechanism of heavy metals in soil. Agricultural Soil Reference Materials, (2):8-19.

[34] Xu, Jiaquan. 1994. The Rule of Migration and Transformation of Injurious Element Chromium in Water Body Environment. Journal of China University of Mining \& Technology, 23(01):53-59.

[35] Yan, Feng. 2010. Environmental Behave of Cr(VI) Uptake and Cr(III) Oxidation as Affected by Soil Properties. Yang Ling: Northwest A\&F Univ..

[36] Yan, Feng \& Liu, Heman \& Liang, Dongli , et al. 2008. Kinetic characteristics of hexavalent chromium apparent adsorption on different soils, Transactions of the CSAE, 24(6):21-25.

[37] Yang, Junxiang \& Lan, Yeqing. 2005. Study on kinetics of chromium (VI) reduction by sulfide. Acta Scientiae Circumstantiae, 25(03):356-360.

[38] Yang, Hongmin \& Yu, Congzheng \& Xie, Hai.2009. Study on Reaction between Hydroxyl Free Radical Oxide $(\bullet \mathrm{OH})$ and $\mathrm{Cr}(\mathrm{III})$. Leather and Chemicals, 26(03):1-5.

[39] Yang, Bin. 2006. Study on the chromium adsorption characteristic and influencing factors of soil. Gui Yang: Guizhou University

[40] Yang, Xiuli. 2011. Establishment and validation of the water-rock intereaction modelling between the Malan loess and Cr (III), Hydrogeology\& Engineering Geology, 38(1):99-102.

[41] Yi, Xiu. 2004. Adsorption Characters and Transformation Rate of Cr(III) in Loessial Soil, Journal of Agro-Environment Science, 23(4):700-704.

[42] Zhao, Kun \& Chai, Liyuan \& Wang, Yunyan, et al. 2006. The Existing Form, Migration and Transformation Laws of $\mathrm{Cr}$ in Water Environment, Industrial Safety and Environmental Protection, 32(08):1-3.

[43] Zhang, Yongliang. 1990. Adsorption and Extraction of Hexavalent Chromium in Soil. Environmental Chemistry, 9(04):43-48.

[44] Zhu, Yuezhen. 1985. Factors Influencing the Migration and Transformation of Chromium in Soil. Acta Pedologica Sinica, (04):390-393. 